\title{
THE OPTICAL LUMINOSITY FUNCTION OF GAMMA-RAY BURSTS DEDUCED FROM ROTSE-III OBSERVATIONS
}

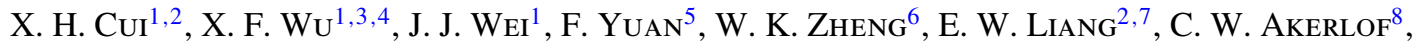 \\ M. C. B. Ashley ${ }^{9}$, H. A. Flewelling ${ }^{10}$, E. GöĞÜş ${ }^{11}$, T. GÜVer ${ }^{12}$, Ü. KıZıloĞLu ${ }^{13}$, T. A. McKaY ${ }^{8}$, \\ S. B. PANDEY ${ }^{14}$, E. S. RyKofF ${ }^{15}$, W. Rujopakarn ${ }^{16,17}$, B. E. SChaEFer ${ }^{18}$, J. C. WheEler ${ }^{19}$, and S. A. Yost ${ }^{20}$ \\ ${ }^{1}$ Purple Mountain Observatory, Chinese Academy of Sciences, Nanjing 210008, China; xhcui@bao.ac.cn, xfwu@pmo.ac.cn, jjwei@pmo.ac.cn \\ 2 National Astronomical Observatories, Chinese Academy of Sciences, Beijing 100012, China \\ ${ }^{3}$ Chinese Center for Antarctic Astronomy, Nanjing 210008, China \\ ${ }^{4}$ Joint Center for Particle, Nuclear Physics and Cosmology, Nanjing University-Purple Mountain Observatory, Nanjing 210008, China \\ ${ }^{5}$ Research School of Astronomy and Astrophysics, The Australian National University, Weston Creek, ACT 2611, Australia; fang.yuan@anu.edu.au \\ ${ }^{6}$ Department of Astronomy, University of California, Berkeley, CA 94720-3411, USA; zwk@ astro.berkeley.edu \\ ${ }^{7}$ Department of Physics and GXU-NAOC Center for Astrophysics and Space Sciences, Guangxi University, Nanning 530004, China; lew@gxu.edu.cn \\ ${ }^{8}$ Department of Physics, University of Michigan, Ann Arbor, MI 48109, USA \\ ${ }^{9}$ School of Physics, University of New South Wales, Sydney, NSW 2052, Australia \\ ${ }^{10}$ Institute for Astronomy, University of Hawaii, 2680 Woodlawn Drive, Honolulu, HI 96822, USA \\ ${ }^{11}$ Sabancı University, Orhanl1-Tuzla, 34956 İstanbul, Turkey \\ 12 Department of Astronomy and Space Sciences, Istanbul University Science Faculty, 34119 Istanbul, Turkey \\ 13 Middle East Technical University, 06531 Ankara, Turkey \\ 14 ARIES, Manora Peak, Nainital 263129, Uttarakhand, India \\ 15 SLAC National Accelerator Laboratory, Menlo Park, CA 94025, USA \\ ${ }^{16}$ Department of Physics, Faculty of Science, Chulalongkorn University, 254 Phayathai Road, Pathumwan, Bangkok 10330, Thailand \\ ${ }^{17}$ Kavli Institute for the Physics and Mathematics of the Universe (WPI), Todai Institutes for Advanced Study, The University of Tokyo, Tokyo, Japan \\ 18 Department of Physics and Astronomy, Louisiana State University, Baton Rouge, LA 70803, USA \\ ${ }^{19}$ Department of Astronomy, University of Texas at Austin, Austin, TX 78712, USA \\ ${ }^{20}$ Department of Physics, College of St. Benedict, St. John's University, Collegeville, MN 56321, USA \\ Received 2014 January 20; accepted 2014 September 12; published 2014 October 16
}

\begin{abstract}
We present the optical luminosity function (LF) of gamma-ray bursts (GRBs) estimated from a uniform sample of 58 GRBs from observations with the Robotic Optical Transient Search Experiment III (ROTSE-III). Our GRB sample is divided into two sub-samples: detected afterglows (18 GRBs) and those with upper limits (40 GRBs). We derive $R$-band fluxes for these two sub-samples $100 \mathrm{~s}$ after the onset of the burst. The optical LFs at $100 \mathrm{~s}$ are fitted by assuming that the co-moving GRB rate traces the star formation rate. While fitting the optical LFs using Monte Carlo simulations, we take into account the detection function of ROTSE-III. We find that the cumulative distribution of optical emission at $100 \mathrm{~s}$ is well described by an exponential rise and power-law decay, a broken power law, and Schechter LFs. A single power-law (SPL) LF, on the other hand, is ruled out with high confidence.
\end{abstract}

Key words: gamma-ray burst: general - methods: statistical

Online-only material: color figures

\section{INTRODUCTION}

Gamma-ray bursts (GRBs) are the most luminous explosions in the universe. Although highly transient, they provide a good laboratory to study astrophysics in extreme conditions. Prompt gamma-ray emission refers to the emission component detected by gamma-ray detectors and is commonly interpreted as emission from internal shocks (e.g., Rees \& Mészáros 1994, 2005; Daigne \& Mochkovitch 1998; Pe'er et al. 2006) or internal magnetic energy dissipation processes (e.g., Usov 1992; Giannios \& Spruit 2006; Zhang \& Yan 2011). Prompt emission is often followed by an afterglow that is the multiwavelength radiation from the external shock produced by interactions between the ejecta from the fireball and the ambient medium (see, e.g., Zhang \& Mészáros 2004 and Gao et al. 2013 for a review).

The identification of the first GRB redshift by Metzger et al. (1997; GRB 970508) revealed the cosmological origin and vast energy release from GRBs, which allows their detection out to extreme distances, e.g., $z \sim 8.2$ (Tanvir et al. 2009; Salvaterra et al. 2009), and possibly at redshifts as high as $\sim 9.4$ (Cucchiara et al. 2011). However, the transient nature of GRBs means that only a limited number have spectroscopic redshifts. This motivates search into correlations between GRB luminosity and various observable parameters in order to derive pseudo-redshifts for GRB events without spectroscopic redshifts (e.g., Norris et al. 2000; Lloyd-Ronning et al. 2002; Firmani et al. 2004; Kocevski \& Liang 2006; Schmidt 2009). There is evidence for potential luminosity evolution in the gamma-ray band (Salvaterra et al. 2012), but the luminosity function (LF) is strongly dependent on the instrumental detection function, which complicates the interpretation of the results.

Following the identification of the first optical counterpart of a GRB in 1997 February 28 (van Paradijs et al. 1997), many statistical studies of optical afterglow (OA) light curves have been carried out, resulting in our current understanding of the general features of the light curve. Dai (2009) compared the cumulative distributions of peak gamma-ray photon fluxes and showed that Swift and BATSE samples come from the same parent population of bursts. Morphological studies of light curves based on statistical analyses of large samples indicate that there are several emission components in the optical afterglow (e.g., Liang \& Zhang 2006; Panaitescu \& Vestrand 2008, 2011; Kann et al. 2010, 2011). Two universal tracks of the late optical 
luminosity light curves have been found (e.g., Nardini et al. 2006, Kann et al. 2006). Kann et al. (2010, 2011) compared the optical light curves of different types of GRBs in the pre-Swift and Swift eras to study the distribution of early luminosities at $43.2 \mathrm{~s}$ in the bursts' rest frame with known redshifts and hostgalaxy extinctions. They found that the luminosity distribution can be approximated by three Gaussians. The typical features of GRB OA light curves comprise an early bump and plateau components (Panaitescu \& Vestrand 2008, 2011; Li et al. 2012; Liang et al. 2013; Wang et al. 2013). Wang et al. (2013) found that a single power law provides a good description of the LF at $10^{3} \mathrm{~s}$. Most of the optical data for these studies, however, were collected from inhomogeneous observations with different instruments. Another issue is that optical observations often only start after the end of prompt gamma-ray emission. The optical LF of GRB afterglows is therefore poorly known since no complete sample within a given threshold is available. The detection of an optical counterpart of a GRB depends on the instrument, exposure time, observation epoch, etc. Therefore, a homogeneous data set from a single instrument, e.g., the Robotic Optical Transient Search Experiment III (ROTSE-III) in this work, can reduce these uncertainties. An analysis of early (e.g., at $100 \mathrm{~s}$ ) and homogeneous data after the onset of the burst is desirable to facilitate the interpretation of the optical LF of GRBs.

Although the Swift satellite has led to an increase in the number of GRBs with good redshift determinations, the sample is still not sufficiently large to directly measure the LF and is affected by various biases. Since long GRBs are associated with the deaths of massive stars, the assumption that the GRB rate traces the star-formation rate (SFR) has been used by many studies to constrain the GRB LF (e.g., Lamb \& Reichart 2000; Choudhury \& Srianand 2002; Natarajan et al. 2005; Daigne et al. 2006). Following this methodology and assuming the most recent SFR determinations, we derive the optical LF of GRBs by fitting the observed ROTSE-III flux distributions at $100 \mathrm{~s}$ after the prompt gamma-ray emission. This allows us to construct the optical LF of GRBs without needing to know their redshifts. The assumption we make is that the global rate of GRB OAs is proportional to the SFR and LF.

In this paper, we take advantage of the large sample of GRBs observed by ROTSE-III to explore the shape of the optical LF at early emission phases. The paper is organized as follows. In Section 2, we present our GRB sample and the method we used to reproduce the flux distribution of the GRBs. Our results are described in Section 3. Section 4 concludes with a discussion. In this paper, we adopt $\Omega_{m}=0.3, \Omega_{\Lambda}=0.7$, and $H_{0}=70 \mathrm{~km} \mathrm{~s}^{-1} \mathrm{Mpc}^{-1}$.

\section{OBSERVATIONAL DATA AND METHODOLOGY}

ROTSE-III is a network of four identical $0.45 \mathrm{~m}$ telescopes distributed around the world to promptly observe OA of GRBs (Akerlof et al. 2003). We use a sample of ROTSE-III data to derive the GRB optical LF. The observed GRB OA rate is assumed to be a convolution of the optical LF with the cosmic GRB rate history. Since the intrinsic LF shape is affected by instrumental sensitivity, we use simulations to determine this effect.

\subsection{ROTSE-III Observations}

We selected our sample of GRBs from ROTSE-III observations between 2005 February and 2011 July. For uniformity, we defined an epoch for brightness measurement to be $100 \mathrm{~s}$ after the burst (with an equivalent exposure time of $5 \mathrm{~s}$ ). A non-detection, namely, a $3 \sigma$ upper limit measurement, is also considered if it meets the following two criteria. First, we only consider GRBs that were triggered by the Swift satellite in order to have a uniform solid angle of sky coverage (see Section 2.2 below). Second, the GRB must have ROTSE-III observations both before and after $100 \mathrm{~s}$, thereby allowing an interpolation to $100 \mathrm{~s}$. We have not included GRB 080319B, the naked-eye GRB, which was observed under inclement conditions with CCD condensation (Swan et al. 2008). For upper limit measurements, we used a transformation factor to allow for different exposure times. Since most of the upper limit measurements were obtained with an exposure of $5 \mathrm{~s}$, we normalized all the longer exposure times (either $20 \mathrm{~s}$ or $60 \mathrm{~s}$ ) to $5 \mathrm{~s}$ exposures. Some GRBs have optical detections in exposures longer than $5 \mathrm{~s}$, but their observed magnitudes are fainter than the equivalent $5 \mathrm{~s}$ limiting magnitude of the instrument (e.g., GRB050401; 8 GRBs in total, which are marked with stars in Table 1). These bursts are considered to be non-detections for the purpose of optical LF construction.

Our final sample consists of $58 \mathrm{GRBs}$ with 18 detections and 40 upper limit measurements. Some of the detections have been published previously (Yost et al., 2007a, 2007b; Rykoff et al. 2009; Yuan et al. 2008; Yuan 2010). For unpublished data, we use the ROTSE-III photometry package (RPHOT; Rykoff et al. 2009) to perform PSF photometry. Since all the ROTSEIII observations were taken unfiltered and the response of the instrument is approximately in the $R_{C}$ band (Rykoff et al. 2009), we adopt $R_{C}$ as our bandpass for photometry.

We take into account and make corrections for extinction in both our Galaxy and the GRB host galaxy. We correct for galactic extinction $\left(A_{V}\right)$ using the values given by Schlafly \& Finkbeiner (2011) as listed in Table 1. We transform the value of $A_{V}$ to $A_{R}$ by applying an average extinction law (Cardelli et al. 1989). GRB $110625 \mathrm{~A}$ is located in a region with very high extinction, $A_{V}=30.29$ (Schlafly \& Finkbeiner 2011), making it difficult to place any constraints on the LF based on this burst, which was therefore excluded from our non-detection sample. We adopt a mean value of $A_{V}=0.2$ (Kann et al. 2010) for the host galaxy extinction. We did not consider the uncertainties involved in assuming a mean $A_{V}$, including the effects of redshift and the change of attenuation law, since it is difficult to quantify the exact values of these effects. Finally, we corrected the flux from the observer frame to the cosmological rest frame using $F(v, t)=\kappa F_{\text {obs }}(v, t)$, where $F_{\text {obs }}(\nu, t)$ is the flux in the observer frame. The parameter $\kappa$ is defined by $\kappa=(1+z)^{\beta_{o}-\alpha_{o}-1}$ (with the convention $\left.F(v, t) \propto v^{-\beta_{o}} t^{-\alpha_{o}}\right)$, where we adopted the spectral index $\beta_{o}=0.75$ and power-law index $\alpha_{o}=1$ for the light curves of the optical afterglows. We list the basic properties of the GRBs, namely, the start and end observed time $\left(t_{\text {start }} \&\right.$ $t_{\text {end }}$ ), coordinates (R.A. \& Dec), Galactic extinction $A_{V}$, and the observed flux $F_{\text {obs }}$ of the GRBs in our samples comprising 18 detections and 40 upper limits, in Table 1.

\subsection{Optical Luminosity Functions}

The observed rate of GRB OAs with peak fluxes between $F_{1}$ and $F_{2}$ is

$$
\begin{aligned}
\frac{d N}{d t}\left(F_{1}<F<F_{2}\right)= & \int_{0}^{z_{\max }} \int_{L\left(F_{1}, z\right)}^{L\left(F_{2}, z\right)} \Phi(L) \\
& \times \frac{R_{\mathrm{GRB}}(z)}{1+z} \frac{\Delta \Omega}{4 \pi} \frac{d V(z)}{d z} d L d z,
\end{aligned}
$$


Table 1

Properties of the ROTSE-III GRB Sample at $100 \mathrm{~s}$ After Trigger

\begin{tabular}{|c|c|c|c|c|c|c|}
\hline RB & $\begin{array}{c}t_{\text {start }} \\
(\mathrm{s})\end{array}$ & $\begin{array}{l}t_{\text {end }} \\
(\mathrm{s})\end{array}$ & $\begin{array}{l}\text { R.A. } \\
\text { (J2000) }\end{array}$ & $\begin{array}{l}\text { Decl. } \\
\text { (J2000) }\end{array}$ & $\begin{array}{c}A_{V} \\
(\mathrm{mag})\end{array}$ & $\begin{array}{c}F_{\text {obs }} \\
\left(\mathrm{erg} \mathrm{cm}^{-2} \mathrm{~s}^{-1}\right.\end{array}$ \\
\hline \multicolumn{7}{|c|}{18 Detected GRBs } \\
\hline 0801 & 21.8 & 10357.0 & $13: 36: 34.6$ & $-21: 55: 48.0$ & 0.255 & 3.22 \\
\hline 1109A & 35.4 & 14534.9 & $22: 01: 15.8$ & $40: 51: 00.0$ & 0.502 & \\
\hline 51111 & 29.4 & 8561.0 & $23: 12: 32.6$ & $18: 22: 01.2$ & & \\
\hline 50605 & 49.4 & 6677.6 & $21: 28: 30.7$ & $-06: 04:$ & & \\
\hline 60729 & 64.5 & 3045.9 & $06: 21: 08.9$ & $-62: 13: 15.6$ & 0.146 & 4.74 \\
\hline 51007 & 27.2 & 15051.9 & 03:05:11.8 & $-50: 29: 45.6$ & 0.054 & 15.40 \\
\hline $30413 \mathrm{~A}$ & 20.4 & 3190.9 & 19:09:12.2 & $-27: 40: 37.2$ & 0.441 & 5.06 \\
\hline 30603B & 23.0 & 18238.0 & $11: 46: 13.0$ & $68: 03: 39.6$ & 0.033 & 17.02 \\
\hline 80607 & 22.0 & 4792.3 & & $15: 54$ & 0.060 & 1.49 \\
\hline 30703 & 33.6 & 3926.1 & $06: 47: 17.3$ & $-63: 12: 39.6$ & 0.192 & 3.91 \\
\hline 0804 & 19.6 & 8442.3 & $21: 54: 42.0$ & $-53: 11$ & 0.043 & 1.83 \\
\hline 0810 & 35.3 & 9643.6 & $23: 47: 07.9$ & $00: 18$ & 0.075 & 4.28 \\
\hline 1008 & 41.9 & 4450.9 & $18: 39: 52.3$ & $-57: 25$ : & 0.252 & \\
\hline 1029 & 86.7 & 3911.4 & 23:07:0 & $-68: 10:$ & & \\
\hline $0418 \mathrm{~A}$ & & & & & & \\
\hline J. & 17.4 & 244 & $11: 5$ & $26: 3$ & 0.063 & 2.07 \\
\hline 618 & 24.7 & 45834.9 & $19: 3$ & $78: 21$ & 0.231 & 18.12 \\
\hline & 27.2 & 6806.5 & $02: 51: 54.7$ & $49: 16: 40.8$ & 0.865 & 10.47 \\
\hline \multicolumn{7}{|c|}{40 Upper Limit GRBs } \\
\hline 050215A & 65 & 2 & $23: 1$ & $49 \cdot 19$ & 0. & 6. \\
\hline & & & & .2 & & \\
\hline & 33.2 & & $16: 3$ & $02: 11$ & 0.177 & 2 \\
\hline & 31.8 & 100.9 & 03: & $-46: 0$ & 0.0 & \\
\hline & 85.7 & & $23: 2$ & $-31:$ & 0.0 & 4.59 \\
\hline & 27.0 & & $04: 5$ & $28:$ & 1.666 & 29.10 \\
\hline $111 \mathrm{~B}^{*}$ & 32.8 & & 19:0 & & 0.297 & 12.95 \\
\hline 60116 & 79.0 & 2 & $05: 3$ & -05 & 0.6 & 7.66 \\
\hline 50614 & 26.8 & 9.2 & $21: 23: 30.5$ & $-53: 01$ & 0.058 & 8.88 \\
\hline $60004 \mathrm{P}$ & 19.3 & 6608.3 & $03: 52: 52.3$ & $-00: 43: 44.4$ & 0.472 & 4.43 \\
\hline $060927^{*}$ & 16.8 & 1768.7 & $21: 58: 11.3$ & $05: 22: 12.0$ & 0.1 & 3.53 \\
\hline 061121* & 21.7 & 1169.5 & $09: 48: 54.7$ & $-13: 11: 16.8$ & 0.121 & 14.13 \\
\hline & & & 23:53:01.0 & & & \\
\hline & 40.8 & & $13: 1$ & & & \\
\hline & 81 & & & & & \\
\hline & 96.7 & & 19: & -32 & 0. & 1 \\
\hline & 44.7 & 99 & 00: & -29 & 0. & 5 \\
\hline 21 & 24.1 & 7 & 21: & -24 & 0.130 & .14 \\
\hline 704 & 94.3 & & 23: & .2 & 4.888 & 50 \\
\hline 0808 & 29.6 & 1839.4 & $00: 27: 02.6$ & 01:10:48.0 & 0.068 & 3.92 \\
\hline 71001 & 50.7 & 1058.9 & $09: 58: 49.7$ & $-59: 45: 46.8$ & 2.356 & 106.7 \\
\hline & & 2981.4 & $23: 40: 15.6$ & $31: 47: 02.4$ & 0.195 & 3.66 \\
\hline 071118 & 83.9 & 1001.2 & $19: 59: 21.4$ & 70:07:48.0 & 0.945 & 8.01 \\
\hline 080229A & 32.8 & 1856.8 & $15: 12: 52.8$ & $-14: 41: 49.2$ & 0.398 & 19.27 \\
\hline 080303 & 20.4 & & $07: 28: 0$ & $-70: 13: 5$ & & \\
\hline 500 & 22.3 & & & & & \\
\hline 80604 & 84.7 & 1833.3 & $15:$ & .2 & 0.1 & 34 \\
\hline 903 & 24.7 & .6 & $05: 4$ & $51: 15$ & 0.560 & 5.62 \\
\hline 080916A & 26.3 & 1043.0 & $22: 25: 09.4$ & $-57: 01: 33.6$ & 0.051 & 32.08 \\
\hline 081121 & 57.1 & 3125.1 & 05:57:07.7 & $-60: 36: 43.2$ & 0.135 & 204.6 \\
\hline 090407 & 40.8 & 1102.7 & $04: 35: 55.0$ & $-12: 41: 02.4$ & 0.180 & 18.96 \\
\hline 090621A & 60.8 & 889.9 & $00: 43: 56.9$ & $61: 56: 16.8$ & 6.025 & 11370 \\
\hline 090709A & 26.6 & 1259.7 & 19:19:46.6 & $60: 43: 40.8$ & 0.242 & 4.19 \\
\hline 090904A & 85.5 & 1076.3 & $06: 43: 25.2$ & $50: 14: 06.0$ & 0.262 & 6.17 \\
\hline 091208A & 30.5 & 1262.4 & 00:01:10.8 & $65: 40: 48.0$ & 4.247 & 461.6 \\
\hline 091221 & 25.8 & 4964.8 & $03: 43: 11.5$ & $23: 14: 34.8$ & 0.568 & 7.46 \\
\hline $100621 \mathrm{~A}$ & 33.0 & & & $-51: 06: 07.2$ & 0.082 & 3.62 \\
\hline $100802 \mathrm{~A}$ & 34.1 & 1776.2 & 00:09:55.7 & $47: 45: 07.2$ & 0.322 & 14.94 \\
\hline $110315 \mathrm{~A}$ & 49.2 & 1323.1 & $18: 36: 49.2$ & $17: 32: 13.2$ & 0.669 & 42.96 \\
\hline 110726A* & 14.0 & 429.0 & 19:06:51.1 & $56: 04: 12.0$ & 0.206 & 10.87 \\
\hline
\end{tabular}

Note. These bursts were moved to the upper limit sample from the detected sample based on the $5 \mathrm{~s}$ limiting magnitude of the instrument. where the factor $(1+z)^{-1}$ is a result of cosmological time dilation, the parameter $\Delta \Omega=1.4 \mathrm{sr}$ is the solid angle covered on the sky by Swift (Salvaterra \& Chincarini 2007; we only consider GRBs that are triggered by Swift), and $d V(z) / d z$ is the comoving volume element. The comoving GRB formation rate is assumed to trace the cosmic SFR as

$$
R_{\mathrm{GRB}}(z)=k R_{\mathrm{SFR}}(z),
$$

where the factor $k$ is a constant. The SFR, $R_{\mathrm{SFR}}(z)$, in units of $M_{\odot} \mathrm{Mpc}^{-3} \mathrm{yr}^{-1}$, is parameterized following Hopkins \& Beacom (2006) as

$$
\log R_{\mathrm{SFR}}(z)=a+b \log (1+z)
$$

with

$$
(a, b)= \begin{cases}(-1.70,3.30), & z<0.993 \\ (-0.727,0.0549), & 0.993<z<3.80 \\ (2.35,-4.46), & z>3.80\end{cases}
$$

The maximum redshift $z_{\max }$ is determined by the Lyman $\alpha$ absorption of the emission in the $R$ band.

In this work, we compare the beaming-convolved LF of GRBs $\Phi(L)$ with four model functions:

(1) a single power law (SPL):

$$
\Phi(L)=\frac{1}{L_{*}}\left(\frac{L}{L_{*}}\right)^{\alpha_{L}}
$$

(2) a broken power law (BPL):

$$
\Phi(L)=\frac{1}{L_{*}}\left[\left(\frac{L}{L_{*}}\right)^{\alpha_{L 1}}+\left(\frac{L}{L_{*}}\right)^{\alpha_{L 2}}\right]^{-1} ;
$$

(3) an exponential rise and power-law decay function (ERPLD):

$$
\Phi(L)=\frac{1}{L_{*}}\left(\frac{L}{L_{*}}\right)^{\alpha_{L}} \exp \left(-\frac{L_{*}}{L}\right) ;
$$

(4) and a Schechter function:

$$
\Phi(L)=\frac{1}{L_{*}}\left(\frac{L}{L_{*}}\right)^{\alpha_{L}} \exp \left(-\frac{L}{L_{*}}\right),
$$

where $\alpha_{L}$ and $L_{*}$ are parameters determined by fitting the observational data.

The observed rate of GRB OAs is governed by the LF $\Phi(L)$ and the GRB formation rate $R_{\mathrm{GRB}}(z)$ based on fitted parameters including the factor $k, \alpha_{L}$, and $L_{*}$. The constant $k$ can be removed by normalizing the cumulative flux distribution of GRBs to $N\left(F_{\min }, F_{\max }\right)$ as

$$
\mathcal{N}(<F)=\frac{N\left(F_{\min }, F\right)}{N\left(F_{\min }, F_{\max }\right)} .
$$

We search for the best model parameters by evaluating the consistency between the cumulative flux distribution of the observed and expected GRBs with the one-sample Kolmogorov-Smirnov (K-S) test. In this test, the maximum value of the absolute difference between two cumulative distribution functions, D-stat, is evaluated with a significance level Prob. A larger value of Prob indicates better consistency. A value of Prob $>0.1$ is generally acceptable to claim statistical consistency, while a value of Prob $<10^{-4}$ rejects the hypothesis of consistency with high confidence. 

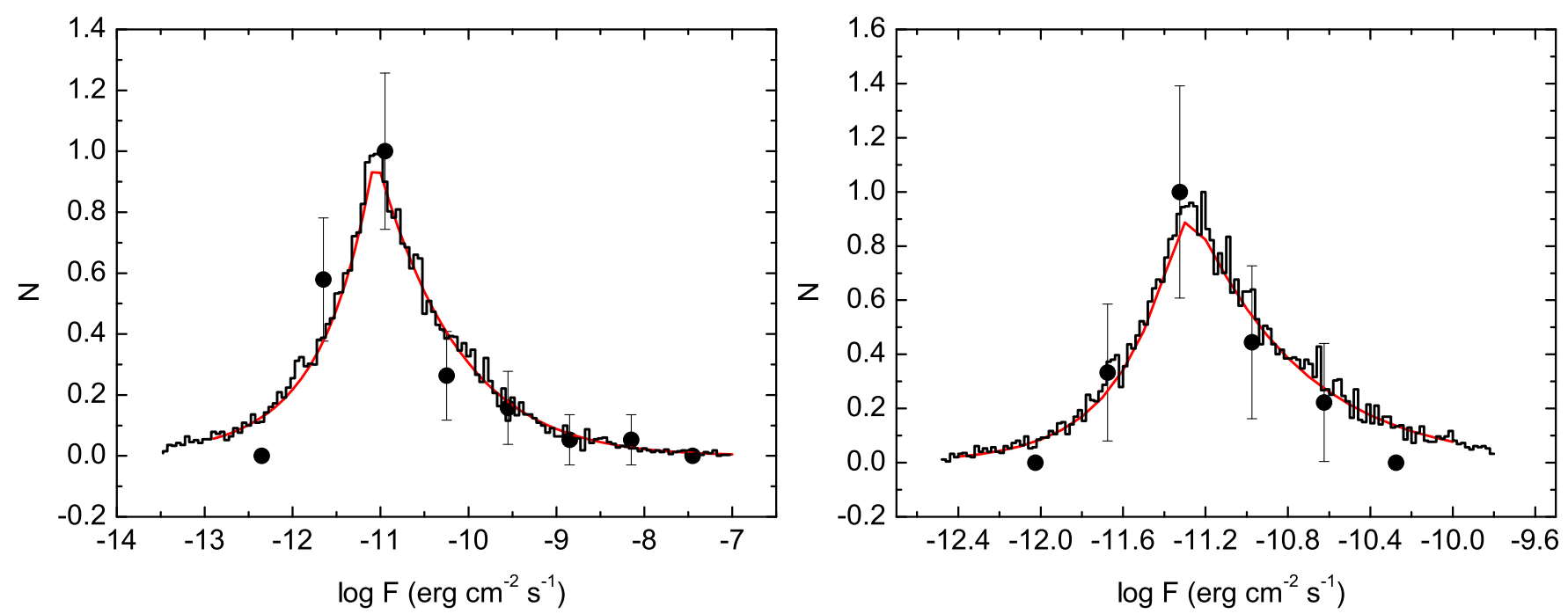

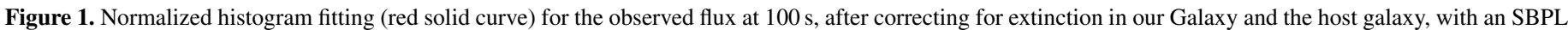

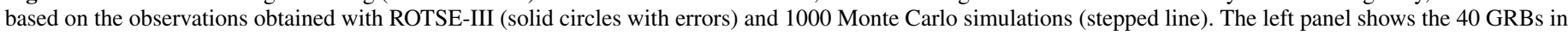
the upper limit sample and the right panel shows the $18 \mathrm{GRBs}$ in the detected sample.

(A color version of this figure is available in the online journal.)

\subsection{ROTSE-III Sensitivity Function}

In order to correct our observed LF for instrumental effects, we performed a simulation based on the number count distribution of the $40 \mathrm{GRBs}$ in our upper limit sample to reconstruct the detection function (i.e., the sensitivity function) of ROTSE-III. The simulation is a four step process that functions as follows. First, we construct a histogram of the flux limit from the 40 GRB limits. Second, a smoothed broken power-law (SBPL) is used to fit this histogram in the observed flux interval,

$$
N=N_{0}\left[\left(\frac{f}{f_{\mathrm{b}}}\right)^{\omega \alpha_{1}}+\left(\frac{f}{f_{\mathrm{b}}}\right)^{\omega \alpha_{2}}\right]^{-1 / \omega},
$$

where the parameter $N_{0}$ is a normalization factor, the parameter $f_{\mathrm{b}}$ is the flux at the break point of the SBPL, the parameters $\alpha_{1}$ and $\alpha_{2}$ are two power-law indices, and the parameter $\omega$ describes the sharpness of the break. The larger the value of the parameter $\omega$, the sharper the break in the SBPL function. Third, we perform a Monte Carlo simulation $(n=1000)$ based on the best-fitting SBPL function. The cumulative distribution of the simulated magnitude limits approximates the actual detection function of the instrument. Finally, the model fitting for this cumulative distribution is applied to find the intrinsic LF of the upper limit sample. A similar simulation for the $18 \mathrm{GRBs}$ in the detected sample is used to reconstruct the detected magnitude distribution. Combining the simulations of the limit and detected sub-samples, a simulated "combined" sample is then applied to constrain the LF obtained from ROTSE-III.

\section{RESULTS}

Figure 1 shows the results of fitting the SBPL function to the simulation histograms for 40 limiting magnitudes (left panel) and 18 detected magnitudes (right panel) with red solid curves. The stepped lines are the Monte Carlo simulations in this figure. The best-fitting parameters, including the normalization factor $N_{0}$, the magnitude $f_{b}$ at the break point, the sharpness factor $\omega$, and the power-law indices $\alpha_{1}$ and $\alpha_{2}$ of SBPL, as described in Equation (10), are presented in Table 2. The null hypothesis for the two groups, i.e., that the data from the observations
Table 2

Fit Results and K-S Test to the Histogram of the Flux $100 \mathrm{~s}$ After Trigger for the Detected and Upper Limit Samples

\begin{tabular}{lccccccc}
\hline \hline Parameter & $N_{0}$ & $m_{b}$ & $\alpha_{1}$ & $\alpha_{2}$ & $\omega$ & Prob & D-stat \\
\hline detected & 0.99 & 11.31 & -20.76 & 41.86 & 5.84 & 0.09 & 0.28 \\
\hline limit & 0.99 & 11.06 & -11.72 & 18.67 & 47.27 & 0.12 & 0.19 \\
\hline
\end{tabular}

obtained with ROTSE-III are from the same population as the simulations, is tested using a K-S test. The maximum distance between the cumulative probability functions of the two groups is $\mathrm{D}$-stat $=0.28,0.19$ with significance levels Prob $=0.09$, 0.12 , respectively. This indicates that one cannot reject the null hypothesis (a common origin of the two samples) at the 5\% significance level, which provides confidence that the simulation based on the best fittings is appropriate in the case that the number of data points may not be large enough to construct the detection function of the instrument. The difference between the detection and limit sub-samples highlights the necessity to consider the detection function in the study of the GRB optical LF.

Figure 2 shows the cumulative distributions of the afterglows fluxes observed by ROTSE-III (solid circles with Poisson error bars in the left panel) and simulated results (stepped lines). The predictions of the flux distribution from the GRB formation rate based on SFR and different LFs are drawn with solid and dashed lines in this figure. The optical LFs with different models (SPL, BPL, ERPLD, and Schechter function) are shown with different colors in the figure. The best-fitting parameters for the models, as well as the results of the K-S test (D-stat, and significance level Prob), are presented in Table 3. Considering that the significance level Prob is also a function of the number of data points, we calculate the value of Prob assuming a simulated number of 100 data points - not the number for the best-fitting selection-for comparison with the observed data. That is, the Prob values do not always correspond to confidence levels and we could not use this value as the standard to select the best fits.

From Table 3, we find that the values of D-stat are smaller for the "combined (simulated)" sample (including 1000 detection simulations and 1000 upper limit simulations) than those for 

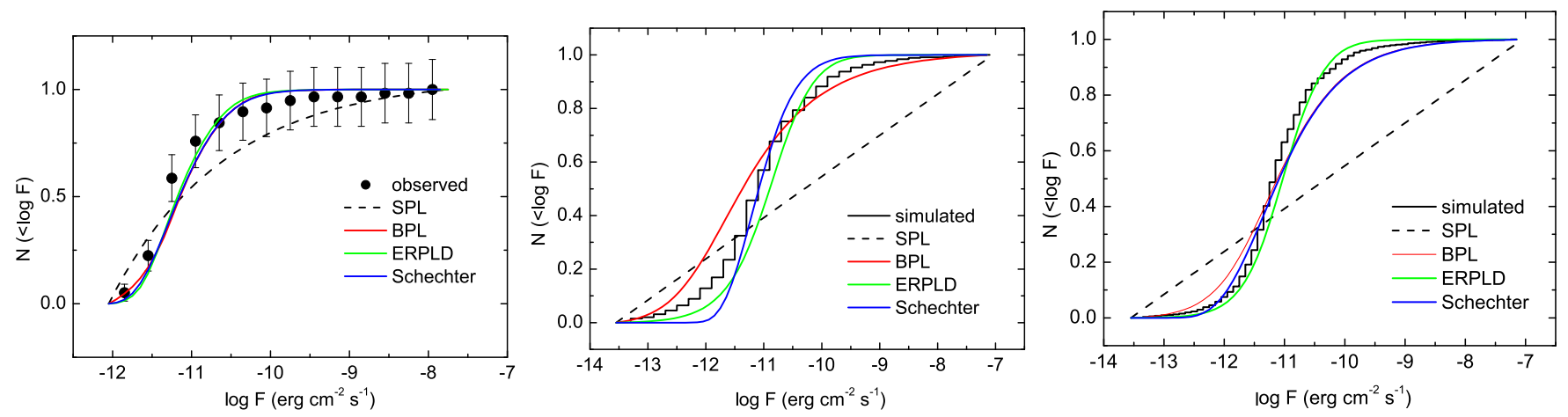

Figure 2. Model fitting results for the cumulative distributions of 58 afterglows observed by ROTSE-III (left panel), simulated sensitivity function (middle panel), and the "combined (simulated)" sample including 1000 detection simulations and 1000 limit simulations. The solid circles with errors labeled in the left panel as "observed" are the afterglows observed by ROTSE-III. The stepped lines are those from simulations. The type of LF is identified by color as described in the text.

(A color version of this figure is available in the online journal.)

Table 3

The Best-fit Models of the Cumulative Distributions of Flux for the Afterglows Observed by ROTSE-III ("obs") and Those for Two Simulations at $100 \mathrm{~s}$ After Trigger

\begin{tabular}{|c|c|c|c|c|}
\hline Model & Parameter & Obs & $\begin{array}{c}\text { Limit } \\
\text { (Simulated) }\end{array}$ & $\begin{array}{r}\text { Combined } \\
\text { (Simulated) }\end{array}$ \\
\hline \multirow{4}{*}{ SPL } & $\alpha_{L}$ & -1.3 & -1.0 & -1.0 \\
\hline & $L_{*}{ }^{\mathrm{a}}\left(10^{46} \mathrm{erg} \mathrm{s}^{-1}\right)$ & 25 & 34 & 32 \\
\hline & D-stat & 0.26 & 0.40 & 0.41 \\
\hline & Prob & $1.4 \times 10^{-3}$ & $1.1 \times 10^{-9}$ & $2.7 \times 10^{-13}$ \\
\hline \multirow{5}{*}{ BPL } & $\alpha_{L 1}$ & 26.0 & 1.4 & 1.6 \\
\hline & $\alpha_{L 2}$ & 0.05 & 0.01 & 0.01 \\
\hline & $L_{*}\left(10^{46} \mathrm{erg} \mathrm{s}^{-1}\right)$ & 14 & 0.9 & 3.4 \\
\hline & D-stat & 0.11 & 0.12 & 0.07 \\
\hline & Prob & 0.52 & 0.06 & 0.20 \\
\hline \multirow{4}{*}{ ERPLD } & $\alpha_{L}$ & 4.9 & 0.01 & 0.4 \\
\hline & $L_{*}\left(10^{46} \mathrm{erg} \mathrm{s}^{-1}\right)$ & 1 & 17 & 8 \\
\hline & D-stat & 0.12 & 0.09 & 0.08 \\
\hline & Prob & 0.72 & 0.05 & 0.25 \\
\hline \multirow{4}{*}{ Schechter } & $\alpha_{L}$ & -4.6 & -3.2 & -1.6 \\
\hline & $\underline{L_{*}\left(10^{46} \mathrm{erg} \mathrm{s}^{-1}\right)}$ & 20 & 13 & 2 \\
\hline & D-stat & 0.10 & 0.08 & 0.07 \\
\hline & Prob & 0.63 & 0.05 & 0.15 \\
\hline
\end{tabular}

Notes. One simulation is for the upper limit sample (detection function). The other is for the "combined (simulated)" sample including 1000 detection simulations and 1000 upper limit simulations. The model functions are SPL, BPL, ERPLD, and Schechter function.

${ }^{a}$ The values from these best fits are not all strongly constrained, e.g., the fits are insensitive to values of $L_{*}$ from 2 to 32 for the "combined (simulated)" sample.

the other two samples, including the "obs," data observed by ROTSE-III, and the"limit (simulated)," the simulated sensitivity function based on the modeled LF excluding the SPL model. This again implies that it is necessary to consider the sensitivity function of an instrument when studying the optical LF of GRBs. For each data sample, the SPL (Equation (5)) LF has the largest value of D-stat among all the LF models, 0.26 for the "obs" sample, 0.40 for the "limited (simulated)" sample, and 0.41 for the "combined (simulated)" sample. This can also be seen from Figure 2 where the SPL model (dashed line) has the largest deviation from the "obs" data and the simulated data (stepped line). We also find that the fits are insensitive to the values of $L_{*}$ for all of the samples. For example, fits are insensitive to values of $L_{*}$ from 2 to 32 for the "combined (simulated)" sample. Excluding the SPL model, the values of D-stat for the "combined (simulated)" sample are in the range [0.07 0.08]. Furthermore, the BPL and Schechter function are also suitable models for the optical LF of GRBs at $100 \mathrm{~s}$. However, the values of D-stat are in the [0.10 0.12] range for "obs" data. For the "limit (simulated)" sample, the Schechter and ERPLD functions better describe the sensitivity function of ROTSE with smaller values of D-stat (0.08 and 0.09), although the BPL function has one more parameter than the others.

\section{DISCUSSION AND CONCLUSIONS}

We construct the optical LFs of GRBs at $100 \mathrm{~s}$ after the burst onset and study their functional form. The sensitivity function of the instrument is carefully considered with simulations and we find it is necessary to take it into account for the study of the LFs of GRBs. We have found that an ERPLDs, BPLs, or Schechter functions are suitable models for the optical LF of GRBs observed by ROTSE III at $100 \mathrm{~s}$. An SPL functional form is excluded as the optical LF based on our GRB sample with high confidence.

We interpret the parameter $k$ in our sample as the ratio of GRBs detected by ROTSE-III in the field of view of Swift to all the bursts happening throughout the sky during the Swift observation time. It is, however, difficult to determine the value of $k$; in the particular case of our study, the K-S test helps eliminate this parameter when finding the best fits by normalizing the cumulative flux distribution of GRBs. An internal shock could produce the emission at the prompt phase and an external shock (reverse shock/forward shock) is thought to be a good candidate for the emission in the afterglow phase. However, the physical reason for the curved LF remains unclear. Kann et al. (2010) interpreted the three Gaussian luminosity distribution as the existence of three "classes" of GRBs. It might be possible that the emission at $100 \mathrm{~s}$ in our work originates from internal processes since they are earlier than those from the afterglow phase.

The optical luminosity was found to increase with increasing prompt energy release (Nysewander et al. 2009; Kann et al. 2010), similar to the X-ray luminosity (e.g., Kouveliotou et al. 2004; Liang \& Zhang 2006; Amati et al. 2007; Gehrels et al. 2008). The plot of the optical luminosity $L_{\text {opt }}$ at $100 \mathrm{~s}$ after the burst onset versus the isotropic energy $E_{\mathrm{iso}, \text { bol }}$ 


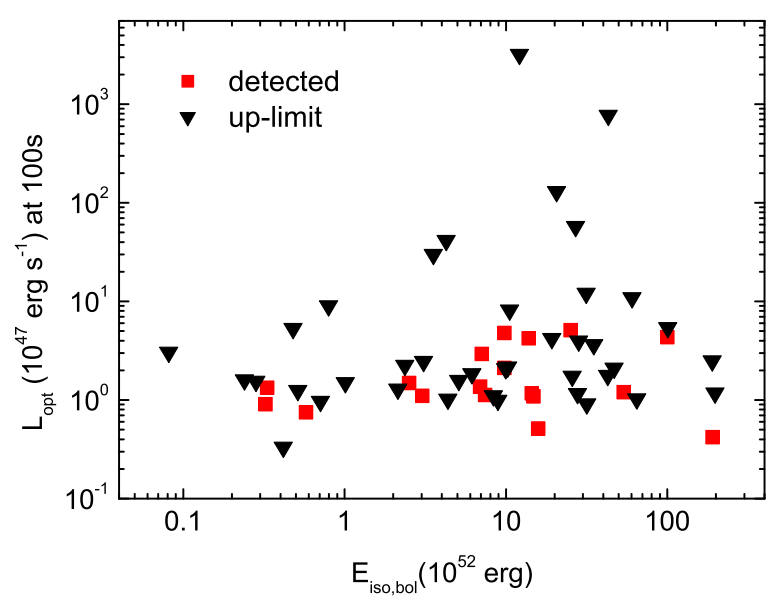

Figure 3. Plot of optical luminosity $L_{\mathrm{opt}} 100 \mathrm{~s}$ after triggering vs. the prompt isotropic bolometric energy $E_{\text {iso,bol }}$ for $58 \mathrm{GRBs}$ reported by ROTSE-III. The black downward pointing triangles are the upper limit reports, and the red squares are optical detections for 18 GRBs.

(A color version of this figure is available in the online journal.)

radiated during the prompt phase of our sample is studied here to compare with previous work. There does not seem to be any trend of luminosity $L_{\mathrm{opt}}$ to energy $E_{\text {iso,bol }}$ in our sample, as shown in Figure 3. The redshifts of GRBs in our sample were taken from Jochen Greiner's Table. ${ }^{21}$ For GRB 110726A, the redshift of 1.036 is based on the only detected absorption line, whereas the upper limit of 2.7 is based on the non-detection of Lyman alpha; we adopt $z=1.036$ as its redshift. For those bursts without redshift measurements (21 GRBs in our sample), we assume redshifts of $z=2$ for the calculation of the luminosity distance of the bursts, since the mean redshift of Swift GRBs has been shown to be close to 2 (e.g., Fynbo et al. 2009). The isotropic bolometric energies $E_{\text {iso,bol }}$ released during the prompt phase of some GRBs in our sample have been calculated by Kann et al. (2010, 2011). For the bursts not included in the work of Kann et al. $(2010,2011)$, we calculated the values $E_{\text {iso,bol }}$ based on Butler's analysis $^{22}$ (Butler et al. 2007) and the GCN report. ${ }^{23}$

Rapid follow-up observations in the optical are critical to understand the physical processes of GRBs. There are quite a few small robotic telescopes, in addition to ROTSE-III, that have been built and installed around the world in order to rapidly search for GRB optical counterparts, e.g., GROCSE (Park et al. 1997), TAROT (Klotz et al. 2009), SkyNet, ${ }^{24}$ WIDGET (Urata et al. 2011), MASTER, ${ }^{25} \mathrm{Pi}$ of the Sky (Burd et al. 2005), RAPTOR (Vestrand et al. 2002), REM (Zerbi et al. 2001), and Watcher (Ferrero et al. 2010). With their large fields of view and fast slewing abilities, these telescopes promise to capture large samples of optical counterparts to accurately constrain the GRB LF at the earliest epoch after burst onset.

We thank the anonymous referee for very constructive suggestions. We thank Z. G. Dai, B. Zhang, and L. P. Xin for helpful discussions. This work was supported by the $\mathrm{Na}$ tional Basic Research Program of China (973 Program, grant No. 2014CB845800 and 2013CB834900) and the National Natural Science Foundation of China (grant No. 11103026,

\footnotetext{
21 http://www.mpe.mpg.de/ jcg/grbgen.html

22 http://butler.lab.asu.edu/swift/

23 http://gcn.gsfc.nasa.gov/gcn/

24 http://skynet.unc.edu/

25 http://observ.pereplet.ru/
}

11322328, and 11025313). X.F.W. acknowledges support by the One-Hundred-Talents Program, the Youth Innovation Promotion Association, and the Strategic Priority Research Program "The Emergence of Cosmological Structures" (grant No. XDB09000000) of the Chinese Academy of Sciences. The research of J.C.W. is supported in part by NSF grant AST-1109801.

\section{REFERENCES}

Akerlof, C. W., Kehoe, R. L., McKay, T. A., et al. 2003, PASP, 115, 132 Amati, L., Della Valle, M., Frontera, F., et al. 2007, A\&A, 463, 913 Burd, A., Cwiok, M., Czyrkowski, H., et al. 2005, NewA, 10, 409 Butler, N. R., Kocevski, D., Bloom, J. S., \& Curtis, J. L. 2007, ApJ, 671, 656 Cardelli, J. A., Clayton, G. C., \& Mathis, J. S. 1989, ApJ, 345, 245 Choudhury, T. R., \& Srianand, R. 2002, MNRAS, 336, L27 Cucchiara, A., Levan, A. J., Fox, D. B., et al. 2011, ApJ, 736, 7 Dai, X. 2009, ApJL, 697, L68 Daigne, F., \& Mochkovitch, R. 1998, MNRAS, 296, 275

Daigne, F., Rossi, E. M., \& Mochkovitch, R. 2006, MNRAS, 372, 1034 Ferrero, A., Hanlon, L., Felletti, R., et al. 2010, AdAst, 2010, 36

Firmani, C., Avila-Reese, V., Ghisellini, G., \& Tutukov, A. V. 2004, ApJ, 611, 1033

Fynbo, J. P., Jakobsson, P., Prochaska, J. X., et al. 2009, ApJS, 185, 526

Gao, H., Lei, W. H., Zou, Y. C., Wu, X. F., \& Zhang, B. 2013, NewA, 57, 141 Gehrels, N., et al. 2008, ApJ, 689, 1161

Giannios, D., \& Spruit, H. C. 2006, A\&A, 450, 887

Hopkins, A. M., \& Beacom, J. F. 2006, ApJ, 651, 142

Kann, D. A., Klose, S., \& Zeh, A. 2006, ApJ, 641, 993

Kann, D. A., Klose, S., Zhang, B., et al. 2010, ApJ, 720, 1513

Kann, D. A., Klose, S., Zhang, B., et al. 2011, ApJ, 734, 96

Klotz, A., Boër, M., Atteia, J. L., \& Gendre, B. 2009, AJ, 137, 4100

Kocevski, D., \& Liang, E. 2006, ApJ, 642, 371

Kouveliotou, C., et al. 2004, ApJ, 608, 872

Lamb, D. Q., \& Reichart, D. E. 2000, ApJ, 536, 1

Li, L., Liang, E. W., Tang, Q. W., et al. 2012, ApJ, 758, 27

Liang, E. W., Li, L., Gao, H., et al. 2013, ApJ, 774, 13

Liang, E. W., \& Zhang, B. 2006, ApJL, 638, L67

Lloyd-Ronning, N. M., et al. 2002, ApJ, 574, 554

Metzger, M. R., Djorgovski, S. G., Kulkarni, S. R., et al. 1997, Natur, 387, 878

Nardini, M., Ghisellini, G., Ghirlanda, G., et al. 2006, A\&A, 451, 821

Natarajan, P., Albanna, B., Hjorth, J., et al. 2005, MNRAS, 364, L8

Norris, J. P., Marani, G. F., \& Bonnell, J. T. 2000, ApJ, 534, 248

Nysewander, M., Fruchter, A. S., \& Pe'er, A. 2009, ApJ, 701, 824

Panaitescu, A., \& Vestrand, W. T. 2008, MNRAS, 387, 497

Panaitescu, A., \& Vestrand, W. T. 2011, MNRAS, 414, 3537

Park, H. S., Ables, E., Band, D. L., et al. 1997, ApJ, 490, 99

Pe'er, A., Mészáros, P., \& Rees, M. J. 2006, ApJ, 642, 995

Rees, M. J., \& Mészáros, P. 1994, ApJL, 430, L93

Rees, M. J., \& Mészáros, P. 2005, ApJ, 628, 847

Rykoff, E. S., Aharonian, F., Akerlof, C. W., et al. 2009, ApJ, 702, 489

Salvaterra, R., Campana, S., Vergani, S. D., et al. 2012, ApJ, 749, 68

Salvaterra, R., \& Chincarini, G. 2007, ApJ, 656, 49

Salvaterra, R., Della Valle, M., Campana, S., et al. 2009, Natur, 461, 1258

Schlafly, E. F., \& Finkbeiner, D. P. 2011, ApJ, 737, 103

Schmidt, M. 2009, ApJ, 700, 633

Swan, H., Yuan, Y., \& Rujopakarn, W. 2008, GCN, 7470, 1

Tanvir, N. R., Fox, D. B., Levan, A. J., et al. 2009, Natur, 461, 1254

Urata, Y., Tashiro, M. S., Tamagawa, T., et al. 2011, PASJ, 63, 137

Usov, V. V. 1992, Natur, 357, 472

van Paradijs, J., Groot, P. J., Galama, T., et al. 1997, Natur, 386, 686

Vestrand, W. T., Borozdin, K. N., Brumby, S. P., et al. 2002, Proc. SPIE, 4845, 126

Wang, X. G., Liang, E. W., Li, L., et al. 2013, ApJ, 774, 132

Yost, S. A., Aharonian, F., Akerlof, C. W., et al. 2007a, ApJ, 669, 1107

Yost, S. A., Swan, H. F., Rykoff, E. S., et al. 2007b, ApJ, 657, 925

Yuan, F. 2010, PhD thesis, Univ. Michigan

Yuan, F., Rykoff, E. S., Schaefer, B. E., et al. 2008, in AIP Conf. Proc. 1065, 2008 Nanjing Gamma-Ray Burst Conference, ed. Y.-F. Huang, Z.-G. Dai, \& B. Zhang (Melville, NY: AIP), 103

Zerbi, F. M., Chincarini, G., Rodonó, M., et al. 2001, in Gamma-Ray Bursts in the Afterglow Era, ed. E. Costa, F. Frontera, \& J. Hjorth (Berlin: Springer), 434

Zhang, B., \& Mészáros, P. 2004, IJMPA, 19, 2385

Zhang, B., \& Yan, H. 2011, ApJ, 726, 90 